\title{
Diskussion zu Sektion IV
}

Die zunehmende Anwendong der Betarezeptorenblocker in der Therapie des Hochdrucks sollte nicht dazu fúhren, dass bei einem bestimmten Patienten eine bewährte Therapie, z.B. mit einem Kombinationspräparat, welches Reserpin enthält, abgesetzt wird. För einige Patienten ist schon 3 X $20 \mathrm{mg}$ Propranolol eine effektive Dosis. Meist wird eine höhere Dosis gebraucht, z.B. 3X40 $\mathrm{mg}$, mehr als $400 \mathrm{mg}$ Propranolol sollte nicht überschritten werden, zumal

Betarezeptorenblocker eine Dosis-Wirkungs-Kurve haben, die nicht linear verläuft, sondern sich im oberen Teil stark abflacht. Für die übrigen Betarezeptorenblocker gelten äquivalente Dosen. Beweis für eine nicht ausreichende Betarezeptorenblockade ist zum Beispiel die Tatsache, dass die Herzfrequenz im Stehen über 72/min ansteigt. Auch die Belastungsherzfrequenz kann als ausreichender Indikator für die Vollständigkeit der Betarezeptorenblockade herangezogen werden.

Falls trotz ausreichender Betarezeptorenblockade der Blutdruck nicht ge-nügend gesenkt wird, sollte zusätzlich ein Saluretikum gegeben werden. Wenn auch das nicht reicht, empfỉehlt sich die zusätzliche Gabe des peripheren Vaso-dilatators Nepresol, also eine Dreifachkombination. Die Entscheidung, ob Betarezeptorenblocker zu einer ausreichenden Blut-drucksenkung geführt haben, lässt sich erst nach Wochen treffen.

Der Ausscheidungsweg der Betarezeptorenblocker spielt bei Niereninsuf-fizienzpatienten eine gewisse Rolle, werden doch einige Betarezeptorenblocker fast vollständig nichtmetabolisiert über die Nieren ausgeschieden. Aber auch die in der Leber metabolisierten Betarezeptorenblocker haben in der Regel aktive Metaboliten, die auch von der Niere ausgeschieden werden müssen. Es sollten also Leber- und Niereninsuffizienz gemeinsam in Rechnung gestellt werden.

Die Diskussion über die Anwendung von Ca-Antagonisten in der Therapie der hypertrophen Myokardiopathie zeigte noch einmal, dass es sich hier um ein im Versuchsstadium befíndliches Therapiekonzept handelt. Bei den nicht sehr erfolgreichen bislang bekannten Therapieversuchen dieser Erkrankung ist dieser neue, vielleicht erfolgversprechende Weg jedoch ohne weiteres gerechtfertigt. Auch Patienten, die in Ruhe keine Obstruktion haben und bei denen durch Katecholamine ein Druckgradient ausgelöst werden kann, wurden mit Ca-Antagonisten behandelt. Eine Differentialtherapie nach dem Motto: mit provo-zierbarem Druckgradient Betablocker, ohne provozierbaren Druckgradient Ca-Antagonisten, wurde nicht durchgeführt; es ergeben sich bislang auch keine Anhaltspunkte dafür, dass sie zu empfehlen ware. 